\title{
La résistance d'entrée de l'eau dans les drains en polychlorure de vinyle annelés
}

\author{
Ch. Lennoz-Gratin CEMAGREF - Antony
}

\begin{tabular}{|c|c|c|c|}
\hline \multicolumn{4}{|c|}{ Notations } \\
\hline$\lambda$ & : hauteur d'une annelure $(\mathrm{m})$ & $R_{p}$ & : rayon de la sphère fictive modélisant une perfo- \\
\hline$B$ & : largeur d'une annelure (m) & & ration $(\mathrm{m})$ \\
\hline$K$ & : conductivité hydraulique du sol $(\mathrm{m} / \mathrm{s})$ & $S$ & : superficie des perforations par unité de lon- \\
\hline$l$ & : largeur d'une perforation $(\mathrm{m})$ & & gueur de drain $\left(\mathrm{cm}^{2} / \mathrm{m}\right)$ \\
\hline$L$ & : longueur d'une perforation $(\mathrm{m})$ & $W$ & : résistance à l'écoulement (s/m) \\
\hline$n$ & $\begin{array}{l}\text { nombre de perforations par rangée et par } m \text { de } \\
\text { drain }\end{array}$ & $\begin{array}{l}W_{e} \\
W_{r}\end{array}$ & $\begin{array}{l}\text { : résistance d'entrée de l'eau dans le drain }(\mathrm{s} / \mathrm{m}) \\
\text { : résistance radiale à l'écoulement }(\mathrm{s} / \mathrm{m})\end{array}$ \\
\hline$N$ & : nombre de rangées de perforations & & \\
\hline$P$ & : nombre total de perforations par mètre de drain & & \\
\hline$q$ & : débit par unité de longueur du drain $(\mathrm{m} / \mathrm{s} / \mathrm{m})$ & $\alpha_{e}$ & : coefficient de résistance d'entrée \\
\hline$R$ & : distance radiale au centre du drain $(\mathrm{m})$ & $\alpha_{e p}$ & : coefficient de résistance d'entrée calculé \\
\hline$R_{0}$ & : rayon du drain $(\mathrm{m})$ & $\alpha$, & : coefficient de résistance radiale à l'écoulement \\
\hline$R_{\text {eff }}$ & : rayon effectif $(\mathrm{m})$ & $\alpha_{t}$ & : coefficient de résistance totale à l'écoulement \\
\hline$R_{i}$ & $\begin{array}{l}\text { : rayon de la sphère d'influence de chaque perfo- } \\
\text { ration }(\mathrm{m})\end{array}$ & $h$ & $\begin{array}{l}\text { charge hydraulique disponible entre le drain et } \\
\text { l'interdrain }(\mathrm{m})\end{array}$ \\
\hline
\end{tabular}

Les drains agricoles utilisés actuellement en France sont des drains annelés en polychlorure de vinyle. Ils sont perforés avec une surface captante minimum de $20 \mathrm{~cm}^{2}$ par mètre; les perforations de petite dimension sont ovalisées et transversales.

Ces tuyaux n'ont pas un fonctionnement hydraulique « idéal». En effet, ils ne se comportent pas comme un matériau totalement perméable car les perforations sont à la fois petites et peu nombreuses : à titre d'exemple, pour un drain de diamètre extérieur $50 \mathrm{~mm}$ (diamètre le plus courant) la surface captante représente moins de $2 \%$ de la surface totale du tuyau. Le passage obligé de l'eau par les perforations représente donc un frein appréciable à l'écoulement. Mais pour améliorer la résistance du tuyau sans en augmenter le coût il est nécessaire de limiter le nombre des perforations; en outre des perforations de trop grand taille impliqueraient une importante pénétration de particules de sol dans la lumière du drain, ce qui risquerait de provoquer un colmatage.

Il est donc nécessaire de trouver un compromis et de définir une surface captante et une taille des perforations optimales. Dans ce but, un terme de «résistance d'entrée " décrivant la gêne à l'écoulement produite par le drain sera défini et on recherchera une formule théorique de cette résistance en fonction des caractéristiques géométriques du drain.

\section{Water entrance resistance into polivinyl-chloride drain tubes}

All the formulae used to determine drain spacing assume the drain to be ideal. Real drain however consists of an impervious wall provided with small openings in order to admit water. The convergence of the streamlines towards these openings gives an entrance resistance, which reduces the hydraulic head available for drainage. On the other hand the drain manufacturers prefer drains with a small opening area because of the price and the strength of materials. This paper presents how to find out the optimum value of the opening area and of the perforations width. 


\section{Notion de résistance d'entrée}

Ernst (1954) exprime la charge nécessaire à l'écoulement de l'eau vers le drain dans un sol homogène et isotrope par la formule :

$$
\Delta h=q \cdot W
$$

$\Delta h$ : charge hydraulique disponible entre le drain et l'interdrain (m)

$q$ : débit par unité de longueur du drain $\left(\mathrm{m}^{2} / \mathrm{s}\right)$

$W$ : résistance à l'écoulement $(\mathrm{s} / \mathrm{m})$.

Le terme de "résistance à l'écoulement " intègre tous les aspects du trajet de l'eau depuis la surface du sol jusqu'au drain. Si l'on considère un écoulement radial vers un "drain idéal " (c'est-à-dire un drain dont les parois n'offrent aucune résistance au passage de l'eau) dans un sol homogène et isotrope, la limite de la région de l'écoulement est un cylindre équipotentiel de rayon $R$; le drain idéal constitue une surface équipotentielle cylindrique de rayon $R_{0}$. $\left(R_{0}<R\right)$ :

$$
\begin{gathered}
\Delta h=H(R)-H\left(R_{0}\right)=\frac{q}{2 \pi K} \ln \frac{R}{R_{0}} \\
W_{r}=\frac{1}{2 \pi K} \ln \frac{R}{R_{0}}
\end{gathered}
$$

$W_{r} \quad$ : résistance radiale à l'écoulement $(\mathrm{s} / \mathrm{m})$

$K \quad$ : conductivité hydraulique du sol $(\mathrm{m} / \mathrm{s})$.

Dans le cas d'un drain réel, l'écoulement vers le drain rencontre une résistance supplémentaire et donc une perte de charge additionnelle. Cette résistance est appelée "résistance d'entrée" et notée « $W_{e}$. DIERICKX (1980) la définit comme étant égale à la différence entre la résistance totale à l'écoulement vers ce drain et la résistance radiale vers un drain idéal de même diamètre $m$.

A partir de la formule (3), on définit un coefficient adimensionnel de résistance $\alpha_{r}=K W_{r}$

$$
\alpha_{r}=\frac{1}{2 \pi} \ln \frac{R}{R_{0}} .
$$

De même, on définit un coefficient adimensionnel de résistance d'entrée : $\alpha_{e}=K W_{e}$.

En 1958, CHILDS et YouNGS montrent que la substitution d'un drain réel à un drain idéal est équivalent à une réduction du diamètre de ce drain théorique. On peut dans les calculs remplacer un drain réel par un drain idéal de plus petit rayon, appelé «rayon effectif » $R_{\text {eff }}$ et directement lié à la résistance d'entrée :

$$
R_{\text {eff }}=R_{0} e^{-2 \pi \alpha_{e}} .
$$

La résistance d'entrée est un facteur qui dépend essentiellement des caractéristiques géométriques du tuyau. Il est intéressant de savoir calculer ou au moins évaluer le coefficient de résistance d'entrée pour chaque type de drain afin de la comparer à une valeur limite pour laquelle la diminution d'efficacité hydraulique du drainage est inacceptable.
Pour évaluer le coefficient de résistance d'entrée dans les drains on a recours généralement à deux méthodes : - la méthode théorique qui tente de résoudre le problème par la voie mathématique et aboutit à des solutions analytiques ou numériques (NIEUWENHUIS 1979, WIDMOSER 1968, ENGELUNG 1953); les formules établies sont relativement longues et compliquées et ne s'appliquent pas aux drains annelés;

- la méthode analogique basée sur l'utilisation des modèles physiques: cuves à solution électrolytique (MONKE 1969, DiERICKX 1980) ou cuves de sable (WESSELING et HoMma 1967, WILLARDSON 1967, BuRGHARDT 1978, LENNOZ 1984); à partir de ces modèles sont établies des formules semi-empiriques pour les drains annelés (DIERICKX, 1980).

Les perforations des drains sont caractérisées par un ensemble de paramètres indépendants: le nombre de rangées de perforation $(N)$, la surface captante $(S)$, la largeur d'une perforation $(l)$, la longueur d'une perforation $(L)$, le nombre de perforations par mètre et par rangée $(n)$. Il est nécessaire d'établir l'influence de chacun de ces paramètres sur la résistance d'entrée afin de pouvoir évaluer, par la suite, les performances hydrauliques d'un drain à partir de ses seules caractéristiques géométriques.

\section{Etude expérimentale}

Une étude expérimentale a été mise en place dans le but d'obtenir des renseignements sur la variation de la résistance d'entrée en fonction des caractéristiques des perforations des drains.

L'expérience est menée avec un massif poreux constitué de billes de verres (diamètre $1,4 \mathrm{~mm}$ ) avec 15 drains différents. Ces 15 drains ont tous un diamètre de $44 / 50 \mathrm{~mm}$; les caractéristiques de leurs perforations sont rassemblées dans le tableau 1.

Tableau 1 : Caractéristiques des drains

\begin{tabular}{rrrrrrr}
\hline $\begin{array}{c}\text { Drain } \\
\mathrm{N}^{*}\end{array}$ & $\begin{array}{c}S \\
\left(\mathrm{~cm}^{2} / \mathrm{m}\right)\end{array}$ & $\begin{array}{c}1 \\
(\mathrm{~mm})\end{array}$ & $N$ & $n$ & $P=N n \begin{array}{c}\alpha_{e} \\
\text { calculé }\end{array}$ \\
\hline 1 & 20 & 0,60 & 8 & 85 & 680 & 0,46 \\
2 & 12 & 0,60 & 6 & 60 & 360 & 0,91 \\
3 & 8 & 0,60 & 6 & 43 & 258 & 1,29 \\
4 & 20 & 0,90 & 8 & 60 & 480 & 0,62 \\
5 & 12 & 0,90 & 6 & 43 & 258 & 1,18 \\
6 & 8 & 0,90 & 3 & 60 & 180 & 1,72 \\
7 & 20 & 1,20 & 4 & 85 & 340 & 0,80 \\
8 & 12 & 1,20 & 3 & 60 & 180 & 1,62 \\
9 & 8 & 1,20 & 3 & 43 & 129 & 2,29 \\
10 & 20 & 1,40 & 6 & 43 & 258 & 1,14 \\
11 & 12 & 1,40 & 4 & 43 & 172 & 1,65 \\
12 & 8 & 1,40 & 3 & 43 & 129 & 2,23 \\
13 & 20 & 2 & 3 & 60 & 180 & 1,47 \\
14 & 12 & 2 & 3 & 43 & 129 & 2,09 \\
15 & 8 & 2 & 4 & 21 & 84 & 3,27 \\
\hline
\end{tabular}


La cuve cubique comportant un drain long d'environ $50 \mathrm{~cm}$ en son centre est alimentée par le haut avec une lame d'eau d'épaisseur constante (fig. 1). Six valeurs de la charge hydraulique à la sortie du drain sont successivement fixées et les débits correspondant sont enregistrés dès que le régime permanent d'écoulement est atteint.

La conductivité hydraulique des massifs étant connue et constante et la validité de la loi de Darcy ayant été vérifiée à partir des valeurs des vitesses d'écoulement atteintes dans le massif, on calcule grâce à des régressions linéaires un "coefficient de résistance totale à l'écoulement $n: \alpha_{t}$. Ce terme intègre une résistance verticale une résistance radiale et la résistance d'entrée dans le drain. La géométrie du massif restant la même dans chaque cas étudié et la conductivité hydraulique étant constante, nous pouvons faire l'hypothèse que toute la variation du terme $\alpha_{t}$ provient de la variation de la résistance d'entrée, seule la géométrie des perforations du drain étant variable dans cette expérience.

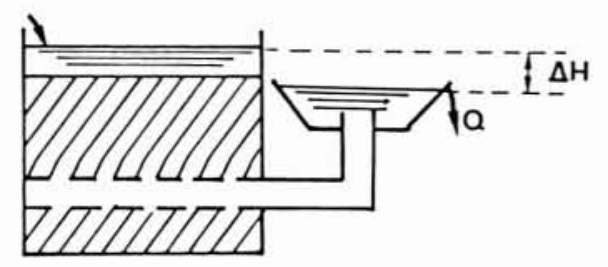

1. Dispositif expérimental.

\section{Etablissement d'une formule théorique du coefficient de résistance d'entrée}

L'expérience réalisée pourra renseigner sur le sens de variation de $\alpha_{e}$ en fonction des dimensions des perforations mais elle ne permet pas de calculer, ni même d'estimer les valeurs du coefficient de résistance d'entrée. C'est pourquoi une formule théorique approximative de $\alpha_{e}$ est recherchée qui permettra après calage sur les résultats expérimentaux de calculer rapidement le coefficient de résistance d'entrée associé à chaque drain.

Le problème est traité en deux dimensions dans le plan perpendiculaire à l'axe du drain. L'écoulement vers le drain dans une cuve cubique est décomposé en des écoulements élémentaires de géométrie simple (fig. 2) :

- écoulement linéaire ;

- écoulement radial ;

- convergence vers les perforations (tenant compte de la position de celles-ci au fond des annelures).

Les perforations sont modélisées par des sphères (et non pas de ellipsoïdes) afin d'aboutir à des solutions mathématiques simples. On aboutit alors à une formule du coefficient de résistance totale à l'écoulement dans la cuve et à une formule de résistance d'entrée théorique.

$\alpha_{e p}=\frac{1}{2 \pi P}\left[\frac{1}{R_{p}}-\frac{1}{R_{i}}-\ln \left(\frac{R_{0}+R_{i}}{R_{0}}\right)\right]+\frac{\lambda}{P L B}$

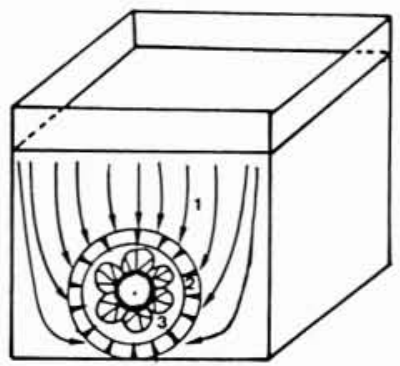

2. Décomposition de l'écoulement vers le drain dans une cuve :
1. écoulement quasi-linéaire
2. écoulement radial
3. écoulement convergent vers les perforations.

$R_{p} \quad$ : rayon de la sphère représentant la perforation $R_{p}=\sqrt{I L / \pi}$

Cette formule est comparée avec la formule semiempirique obtenue par DIERICKX (1980) en utilisant l'analogie électrolytique :

$$
\begin{aligned}
\alpha_{e}=\frac{c}{2 \pi^{2} R_{0}} \ln \frac{2 c}{\pi B} & -\frac{c}{4 \pi R_{0}}+\frac{c \lambda}{N L B}- \\
& -\frac{c}{\pi N L} \ln \sin \frac{\pi l}{2 B}-\frac{1}{2 \pi} \ln \frac{R_{0}}{R_{0}^{\prime}}
\end{aligned}
$$

c : distance entre 2 perforations sur une même génératrice

$R_{0} \quad$ : rayon extérieur maximal du drain

$R_{0}^{\prime} \quad$ : rayon extérieur minimal $\left(R_{0}^{\prime}=R_{0}-2 \lambda\right)$

La formulation est relativement différente mais les applications numériques font état de résultats quasiment identiques.

\section{Résultats et commentaires}

A partir des résultats de l'expérience en cuve, des courbes sont tracées avec le débit en ordonnée et la perte de charge totale en abscisse (fig. 3). On détermine alors par régression linéaire pour chaque drain un coefficient de résistance totale à l'écoulement. Une analyse en composantes principales permet alors de relier $\alpha_{t}$ aux différents paramètres descriptifs du drain. Il ressort de cette analyse que la résistance d'entrée dépend d'abord du nombre total de perforations par mètre de drain et ensuite de la surface captante ; par contre elle dépend assez peu de la largeur des perforations. On retrouve les mêmes informations en utilisant la formule (6) avec les caractéristiques géométriques des drains expérimentaux (tableau 1: $\alpha_{e}$ calculé).

Afin d'augmenter les performances hydrauliques des drains, il est souhaitable de diviser au maximum la surface captante, c'est-à-dire de préférer des perforations petites et nombreuses à des perforations de grande taille et moins nombreuses.

A l'issue de cette étude, les principaux résultats acquis concernent la variation de la résistance d'entrée en fonction des caractéristiques géométriques des perfora- 


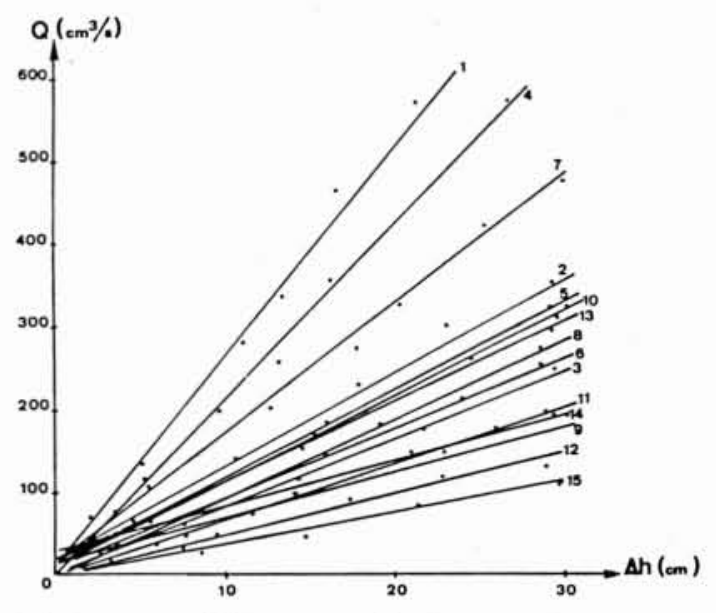

3. Relation entre débit et perte de charge totale pour tous les drains testés.

tions des drains : le coefficient de résistance d'entrée varie en sens inverse de la surface captante et du nombre de perforations. D'un point de vue pratique, on retiendra essentiellement que le minimum de résistance d'entrée est obtenu pour une fragmentation maximale de la surface captante.

Grâce à un modèle analytique simple calé sur des expériences de laboratoire, une formule a été déterminée qui permet de calculer le coefficient de résistance d'entrée pour un drain annelé, en connaissant ses caractéristiques géométriques.

La valeur limite souvent admise pour le coefficient de résistance d'entrée (CROS, 1973) est comprise entre 1 et 1,5 , ce qui correspond à une perte de charge hydraulique de $0,3 \mathrm{~m} / \mathrm{j}$ pour un débit de projet de $1,5 \mathrm{l} / \mathrm{s} / \mathrm{ha}$ et un écartement entre drains de $10 \mathrm{~m}$ ). Cette valeur limite est atteinte avec un nombre de perforations égal à 250 par mètre de drain (avec des performations de $5 \mathrm{~mm}$ de longueur et de $0,6 \mathrm{~mm}$ de largeur au minimum).

La norme française concernant les performations des drains indique des valeurs minimales de la surface captante et de la largeur des perforations et n'impose pas de nombre de perforations minimum. Les valeurs normalisées $S \geqslant 20 \mathrm{~cm}^{2} / \mathrm{m}$ et $0,6 \leqslant 1 \leqslant 2 \mathrm{~mm}$ correspondent à un minimum de 250 perforations et sont compatibles avec les valeurs limite de la résistance d'entrée.

L'étude fine de la résistance d'entrée est motivée en partie par une amélioration éventuelle des performances hydrauliques des réseaux de drainage. Actuellement les formules de dimensionnement des réseaux considèrent le drain comme idéal et ne tiennent pas compte de la perte de charge d'entrée. On peut envisager d'introduire des termes concernant l'écoulement dans l'immédiat voisinage du drain dans les équations du drainage afin d'affiner les calculs des réseaux et d'optimiser le rendement hydraulique.
Mais l'intérêt majeur de la notion de résistance d'entrée concerne son influence sur les phénomènes de colmatage minéral des drains. C'est pourquoi il est nécessaire de comprendre les relations existant entre le coefficient de résistance d'entrée d'un drain et les gradients hydrauliques survenant à l'interface entre le sol et ce drain car ces gradients hydrauliques ont un effet direct sur la déstabilisation du sol et le passage des particules dans la lumière du drain. Une étude approfondie de ces phénomènes permettra de fixer une valeur limite du coefficient de résistance d'entrée admissible pour les drains dans un sol donné et ainsi de choisir les caractéristiques des perforations à prévoir en fonction de l'utilisation du drain.

\section{Bibliographie}

BURGHARDT, W., 1978. Die Wasserbewegung zum Dränrohr in Abhängigkeit von Richtung und Stärke der Anströmung, Rohrfüllung, Rohrdurchmesser und Vollfilterung., Z. $f$. Kult. und Flub., 19, 17-25.

Childs, E. C., Youngs, E. G., 1958. The nature of drain channel as a factor in the design of a land drainage system. Jour. Soil Sci., 9, 2, 316-331.

Cros, P., 1973. Les essais de tuyaux de drainage effectués par le CTGREF. Rapport particulier. Journées Nationales d'Etudes, Orléans, AFEID.

DIERICKX, W., 1980. Electrolytic analogue study of the effects of openings and surrounds of various permeabilities on the performance of field drainage pipes. Communication of the National Institute of Agricultural Engineering, Merelbeke, Belgique, $\mathrm{N}^{*} 77,1-238$.

ENGELUND, F., 1953. On the laminar flows of ground water through homogeneous sand. Trans. Danish Acad. Tech. Sci., A.T.S.3, 1-105.

ERNST, L. F., 1954. Het berekenen van stationaire grondwaterstromingen, welke in een station en Bodemk. Inst. T.N.O. Gröningen, 1-55.

Lennoz-Gratin, C., 1984. Drains en P.C.V. annelés : influence de la taille des perforations sur les écoulements et l'interface sol/drain. Mémoire de DEA CEMAGREF/USTL, 1-101.

MONKE, E. J., 1969. A study of water flow patterns near subsurface drains. Ph. D. Thesis University of Illinois, 1156.

Nieuwenhuis, G., Wesseling, J., 1979. Effect of perforation and filter material on entrance resistance and effective diameter of plastic drain pipes. Agric. Water Man., 2, 1 9.

WESSELING, J., HOMMA, F., 1967. Entrance resistance of plastic drain tubes. Neth. J. Agric. Sci., 15, 170-182.

WidMoser, P., 1968. Der Einfluss von Zonen geänderter Durchlässigkeit im Bereich von Drän-und Brunnenfilterrohren. Schweizerishe Bauzeitung 86, 9, 135-144.

WillaRDSON, L. S., 1967. Water entry into partially full subsurface drains. Ph. D. Thesis Ohio State University, Columbus, 1-194. 